\title{
Performance Evaluation of IPTV Over Wireless Home Networks
}

\author{
Tiantian Guo, Member, IEEE, Chuan Heng Foh, Senior Member, IEEE, Jianfei Cai, Senior Member, IEEE, \\ Dusit Niyato, Member, IEEE, and Eric W. M. Wong, Senior Member, IEEE
}

\begin{abstract}
The emergence of Internet Protocol Television (IPTV) has brought potentials to revolutionize personal entertainment. Streaming TV content over the highly pervasive wireless networks allows easy access to personalized entertainment. Focusing on wireless home entertainment which is one of the main driving forces of IPTV development, we develop a Markovian framework that investigates several important issues related to network capacity and streaming quality in an IEEE 802.11e enabled wireless home network. The Markovian framework captures not only the IEEE 802.11e MAC protocol performance, but also the statistical characteristics of IPTV media streams. The inclusion of these two key descriptions allows our model to be practically used in wireless home network planning and design. To deal with the complexity in the model, we apply the efficient Matrix Geometric approach to obtain numerical results. We further perform simulations with real IPTV traffic to not only validate our analytical results, but also obtain further insight to the performance.
\end{abstract}

Index Terms-IEEE 80211e wireless home networks, Internet Protocol Television (IPTV), performance evaluation.

\section{INTRODUCTION}

$\mathbf{T}$ HE high penetration of wireless home networks has encouraged network applications in the home environment. One of the exciting applications for wireless home networks is Internet Protocol Television (IPTV) which aims to stream TV contents over the Internet. The ability to stream TV contents over wireless Internet not only allows TV contents to reach an even wider range of devices, but also offers great flexibility in time and space control for personal enjoyment.

The current popular wireless home networks use the wireless technologies specified in the IEEE 802.11 standards. The IEEE 802.11 working groups specify a series of wireless communication technologies and medium access control (MAC) protocols that keep the advancement of wireless local area networks (WLANs). Some important standards related to this study include the IEEE $802.11 \mathrm{~b}$ standard specifying the operation of up to $11 \mathrm{Mbps}$ data rate on the $2.4-\mathrm{GHz}$ wireless channel

Manuscript received February 21, 2011; revised April 13, 2011; accepted April 14, 2011. Date of publication May 05, 2011; date of current version September 16, 2011. The associate editor coordinating the review of this manuscript and approving it for publication was Dr. Z. Jane Wang.

T. Guo, C. H. Foh, J. Cai, and D. Niyato are with the Center of Multimedia and Network Technology, School of Computer Engineering, Nanyang Technological University, Singapore (e-mail: guo_tiantian3002@ pmail.ntu.edu.sg; aschfoh@ntu.edu.sg; asjfcai@ntu.edu.sg; dniyato@ntu.edu.sg).

E. W. M. Wong is with the Department of Electronic Engineering, City University of Hong Kong, Hong Kong, China (e-mail: eeewong@ @ityu.edu.hk).

Color versions of one or more of the figures in this paper are available online at http://ieeexplore.ieee.org.

Digital Object Identifier 10.1109/TMM.2011.2150208
[1], the IEEE $802.11 \mathrm{~g}$ standard enhancing the operation to 54 Mbps data rate on the 2.4-GHz wireless channel [2], the IEEE 802.11e standard specifying the enhanced distributed channel access (EDCA) to provide quality-of-service (QoS) support to the IEEE 802.11 WLANs [3], and the recently standardized IEEE $802.11 \mathrm{n}$ standard enhancing the operation to $100 \mathrm{Mbps}$ data rate [4]. With these constant efforts of enhancements, the IEEE 802.11 WLANs have prepared themselves not only to provide wireless Internet access for best effort (BE) traffic, but also to deal with future network applications with high demand in bandwidth and QoS such as multimedia-rich applications [5], [6].

As we witness the advancements in WLANs in the last decade, we also see increasing demand of high-quality media from the traditional low-resolution media to the current DVD quality media, the emerging HDTV quality media, and the potential new arrival of 3-D TV media. IPTV, in which video content is digitized and sent to individual receivers as IP packets, is adopted for most recent media development. IPTV media can be accessed on multipurpose devices instead of traditional TV devices with some dedicated functions. The content of IPTV can be personalized and accessed from locations where Internet connectivity is available. Although a widely-acceptable IPTV standard is not available yet [7], the current popular coding schemes for IPTV include H.264 advanced video coding (AVC), H.264 medium grained scalability coding (MGS), and H.264 scalable video coding (SVC). The rapid evolution of IPTV media in recent years has prompted the urgency in the investigation of the WLAN support. The investigation of the WLAN support for IPTV media is especially important for the IEEE 802.11 WLAN due to its employment of contention-based operation for both channel access and QoS support. The mechanism of the contention-based operation in IEEE 802.11 WLAN gives rise to uncertainties in the efficiency of channel access and effectiveness of QoS support.

As demonstrated in our previous work in [8] focusing on the IEEE 802.11 MAC protocol performance analysis, significant differences in performance exist between a more precise and a less detailed model for the description of the protocol service behavior and the source arrival models. As discussed in [8], the service time distribution of the IEEE 802.11 MAC protocol can be modeled appropriately by Erlang distribution. This permits the construction of a Markovian framework to study the performance of the IEEE 802.11 WLAN under traffic arrivals which can be described by a Markovian arrival process (MAP) [8], [9]. Specifically, it motivates us to design an accurate model for evaluating IPTV performance over WLANs. The contributions of this paper are twofold. 
- Firstly, we present a Markovian framework that investigates the performance of IPTV streaming over the QoS-enabled IEEE 802.11e WLANs. Different from our earlier work [8] which focuses on the proposal of a Markovian framework to describe the service behavior of IEEE 802.11 MAC protocol, this paper considers the IEEE 802.11e network (with QoS feature) as well as the IPTV traffic, where we develop an equivalent MAP to describe the IPTV traffic arrivals and an appropriate phase-type service time distribution to model the EDCA service behavior. To the best of our knowledge, this is the first Markovian framework that can capture the detailed behaviors of IPTV and the IEEE 802.11e EDCA.

- Secondly, to deal with the complexity added by capturing detailed behaviors of IPTV and the IEEE 802.11e EDCA in a Markovian framework which produces more representative performance to the studied scenario, we use the efficient Matrix Geometric approach for the numerical computation.

With the developed Markovian framework, we study the channel capacities (in terms of the number of simultaneous IPTV streams) of various popular IEEE 802.11 versions with the IEEE 802.11e QoS support. We perform simulation experiments to validate our modeling approach and study the performance with aggregated realistic inputs using real video traffic.

The rest of the paper is organized as follows. In Section II, we discuss the related work on the performance evaluation of IPTV over wireless home networks. In Section III, we introduce a typical scenario of IPTV over wireless home networks. In Section IV, we present our developed Markovian framework, describing the modeling of IPTV traffic arrivals and IEEE 802.11e service behavior. In Section V, we show the numerical and simulation results emphasizing the system capacity and the performance characteristics especially under heavy load conditions. Finally, important conclusions are drawn in Section VI.

\section{RELATED WORK}

The importance of developing understanding of the performance of IPTV over the wireless Internet has been highlighted in several recent works (see [10]-[14] and the references therein). Yang et al. [10] claimed that IPTV, as the killer application for the next-generation Internet, needs further investigation and research on detailed performance evaluations in WLANs. Such performance studies can largely be conducted by either empirical experimentation, simulation experimentation, or system modeling.

In [11], Djama and Ahmed evaluated the packet losses and the perceived quality of the TV service using an experimental test-bed. The results obtained from the test-bed well validate the algorithms proposed in [11]. However, the test-bed based performance evaluation suffers from time-consuming realization and complex setup.

Du and Zhang [12] proposed an efficient model to study the statistical QoS guarantees, in terms of effective bandwidth/capacity and delay-bound violation probability, for multi-layer video transmission over wireless fading channels. However, the multi-layer video arrival process was only managed by separate queues, which did not take the statistical characteristics of video traffic into consideration.

Shihab et al. [13], [14] investigated the performance of IPTV packet loss caused by buffer overflow in several home network scenarios including wired link with a constant data rate, single hop wireless link with a variable data rate, and a multi-hop wireless path. For the arrival process, an on-off traffic source was used to describe the video source. For the service process, an exponential distribution was assumed for the service time distribution of the considered data links. The use of an on-off arrival model for video sources and an exponential service time distribution for complex network protocol behaviors such as the IEEE 802.15 .3 or the IEEE 802.11 MAC protocols greatly limits the practicability of the analytical results. Li and Pan [15] proposed an extended two-dimensional Markov-chain model to analyze the throughput of IEEE 802.11 multi-hop wireless networks, where the error-prone channel, non-persistent traffic, post-backoff stage, and finite retry limit were taken into account. The throughput analysis provides the upper-bound throughput performance of video streaming. However, the considered traffic is a general non-persistent traffic which lacks description on video characteristics.

\section{SCENARIO}

The tremendous growing of the IEEE 802.11 WLANs deployed in the home environment in recent years has encouraged the use of IP networks for various traditional and new network applications, such as networking of home appliances, home security, and also IPTV. There are, however, challenges in using a single common wireless home network for home network applications with increasing demands in bandwidth and QoS requirements. The shared wireless channel architecture in WLANs introduces a bottleneck in the network and the employment of contention-based MAC protocol poses uncertainty in the efficiency of the WLANs. These problems have amplifying effect especially in multimedia applications such as IPTV as they demand high and steady bandwidth to provide adequate operations. Anticipating the increasing demand for personal entertainment in the home environment, IPTV will be the next key application in wireless home networks. To develop a theoretical understanding, this paper studies the performance of IPTV streaming over wireless home networks.

Fig. 1 illustrates our considered scenario of IPTV streaming over wireless home networks. Factoring the potential application of personal entertainment in wireless home networks, we consider a scenario of simultaneous IPTV streams in the wireless home network environment, where different IPTV traffic packets are transmitted by unicast to different users. We believe that the IPTV service that offers personalized entertainment is likely to rely on unicast transmission heavily, which is different from the traditional broadcast of TV contents. On the other hand, in terms of system capacity, the analytical model developed in this paper for unicast can also be used to provide estimation for the case of multicast transmission, where the video stream being multicast can be seen as one unicast video stream.

Additionally, the considered wireless home network also carries some amount of BE traffic generated by other network applications. All IPTV users are equipped with a wireless set-top 


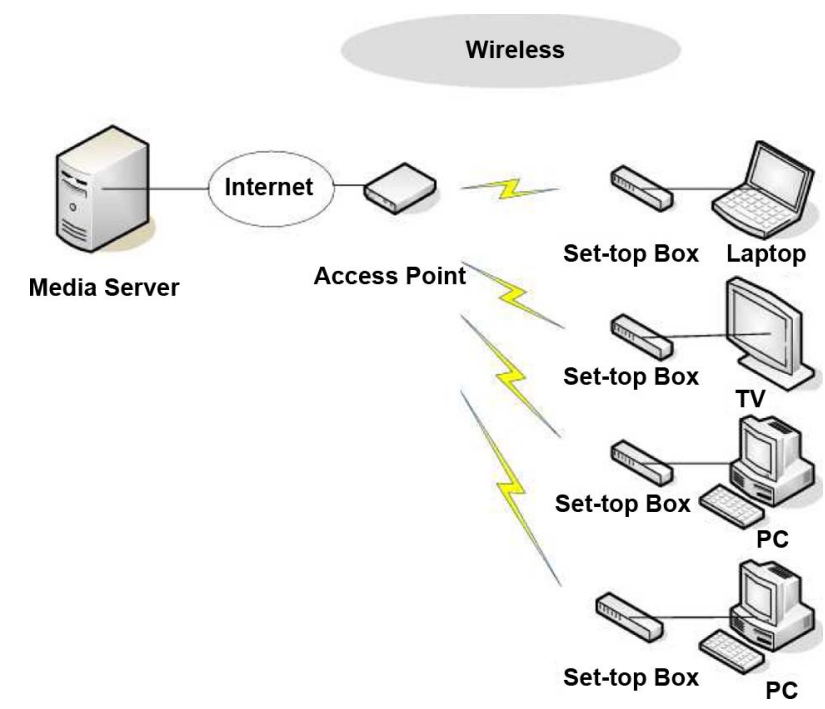

Fig. 1. Scenario example of IPTV over wireless home networks.

box to communicate with a media server on the Internet via the access point (AP) over the wireless home network. In this setup, the AP acts as a relay that forwards the received IPTV streams to all IPTV users over a wireless link.

Given the robust first mile access network and high-speed connectivity between the Internet and a wireless home network, it is expected that streaming of IPTV will find transmission bottleneck at the wireless link within the wireless home network. We assume that the AP enjoys a 100 BASE-T connectivity to the Internet. Our scenario considers various wireless technologies that offer $11 \mathrm{Mbps}, 54 \mathrm{Mbps}$, and $100 \mathrm{Mbps}$ physical data rates, with the IEEE 802.11e EDCA QoS implementation.

The IPTV transmission process can be described as follows. An IPTV user tunes to a particular program where the set-top box makes a request accordingly to the media server. The media server then starts to stream the IPTV traffic over the Internet and the wireless home network to the IPTV user. The IPTV traffic is packetized using RTP/UDP/IP protocols. The IPTV traffic is typically encoded with a pattern of one I frame followed by many $\mathrm{P}$ frames or $\mathrm{B}$ frames to form a group of pictures (GoP) [16]. Each frame is encoded and packetized into one video packet in the application layer which may be subjected to fragmentation in the lower layers. At the receiver side, each frame is decoded from received packets. In the event of a packet loss, not only the frame within the lost video packet is lost, but also the subsequent frames that rely on the lost video frame to decode in the same GoP are considered lost. A lost frame is then replaced by the previously decoded frame for error concealment.

\section{MODEL DESCRIPTION}

Following our previous modeling approach in [8], we develop a Markovian framework to model the described scenario. Precisely, we use a particular MAP to describe the aggregated IPTV streams and a phase-type distribution to describe the service time distribution of the IEEE 802.11e with a certain data rate. In the following subsections, detailed models for the arrival and service processes are described.

\section{A. Traffic Arrival Model}

Our scenario consists of two types of traffic which are IPTV traffic and best effort data traffic. We use two different arrival models to describe these two types of traffic.

For the IPTV traffic arrival model, we consider the statistical characteristics of the IPTV traffic. From the point of view of the AP, the arrival of an IPTV packet is the event that an IPTV packet has arrived at the AP from the Internet ready to be delivered to the intended end user via the wireless link. Given a particular IPTV content, the content is encoded and packetized into a stream of IP packets, each of which is encapsulated into an IEEE 802.11 data frame. Adapting a common traffic shaping control at the video encoding process and ignoring the encoding processing time, it is adequate to describe this IP packet stream using a variable bit rate (VBR) traffic model. One appropriate VBR traffic model is Erlang distribution that specifies the distribution of the inter-arrival time [9].

An Erlang distribution can be characterized by two parameters, namely the number of exponential phases $k$ and the exponential rate $\lambda$. These two parameters control the mean and variance of the distribution, where the mean of an Erlang distribution is $k / \lambda$ and the variance is $k / \lambda^{2}$. By measuring the mean and variance of the packet inter-arrival time of an IPTV traffic trace, we can calculate the corresponding Erlang distribution parameters, $k$ and $\lambda$.

In modeling of an aggregated IPTV traffic stream formed by $n$ different IPTV traffic streams, we again use Erlang distribution for the inter-arrival time distribution. The two parameters, $k$ and $\lambda_{v}$, of the Erlang distribution are now fit based on the aggregated traffic. The arrival rate $r_{v}$ (in bps) of the IPTV traffic can then be calculated as

$$
r_{v}=\frac{L_{v} \cdot \lambda_{v}}{k}
$$

where $L_{v}$ is the average IPTV packet length.

Our model also considers some amount of BE traffic transmission in the network. We use a simple model of Poisson process to describe the $\mathrm{BE}$ arrival process. To appropriately model BE traffic transmission in the IEEE 802.11 WLANs, the uplink BE traffic and the downlink BE traffic are modeled separately. We use $\lambda_{p_{a}}$ and $\lambda_{p_{s}}$ to describe the arrival rates of the downlink BE traffic sent by the AP, and the aggregated uplink BE traffic sent by all stations, respectively.

\section{B. IEEE 802.11e Service Model}

In this subsection, we present a phase-type model that describes the IEEE 802.11e MAC protocol service behavior. We have studied the IEEE 802.11 MAC protocol in our earlier work, where we illustrate the use of Erlang distribution to describe the service time distribution of the IEEE 802.11 MAC protocol [8]. Precisely, our study shows that for the IEEE 802.11 MAC protocol service process, its service time for each packet follows Erlang distribution. Using Bianchi's model [17], we can determine the average service time for a packet given a particular number of active stations in the network.

To deal with the IEEE 802.11e MAC protocol that features the QoS support, a saturation throughput analysis of the IEEE 802.11e MAC protocol is needed. In [18], we extended 
TABLE I

State Transition for the Birth Process From CurRent State $\left(k_{v}, i, j, k_{p_{s}}, k_{p_{a}}\right)$

\begin{tabular}{|c|c|c|c|}
\hline Condition & $\begin{array}{l}\text { Transition rate } \\
\text { (packets/s) }\end{array}$ & Next state & Description \\
\hline$\left[\left(k_{p_{a}}=0\right) \&\left(k_{p_{s}}=0\right) \&\left(k_{v}=0\right)\right] \&(i=\mathrm{I}-1) \&(j=0)$ & $\lambda_{v}$ & $(1,0, J-1,0,0)$ & $\begin{array}{l}\text { One IPTV packet has completed its arrival } \\
\text { process and enters the first service phase } \\
\text { when there is no packet in the buffer. }\end{array}$ \\
\hline$\left(k_{v}<V\right) \&(i<\mathrm{I}-1)$ & $\lambda_{v}$ & $\left(k_{v}, i+1, j, k_{p_{s}}, k_{p_{a}}\right)$ & $\begin{array}{l}\text { One IPTV packet has transited to the next } \\
\text { Erlang phase but yet to complete an } \\
\text { arrival process. }\end{array}$ \\
\hline$\left(k_{v}<V-1\right) \&(i=\mathrm{I}-1)$ & $\lambda_{v}$ & $\left(k_{v}+1,0, j, k_{p_{s}}, k_{p_{a}}\right)$ & $\begin{array}{l}\text { One IPTV packet has completed its arrival } \\
\text { process and enters the first service phase. }\end{array}$ \\
\hline$k_{p_{s}}<P S-1$ & $\lambda_{p_{s}}$ & $\left(k_{v}, i, j, k_{p_{s}}+1, k_{p_{a}}\right)$ & A BE packet from a station has arrived. \\
\hline$k_{p_{a}}<P A-1$ & $\lambda_{p_{a}}$ & $\left(k_{v}, i, j, k_{p_{s}}, k_{p_{a}}+1\right)$ & A BE packet from the AP has arrived. \\
\hline
\end{tabular}

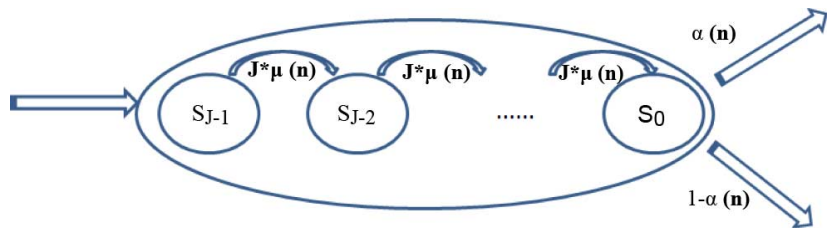

Fig. 2. Phase-type model for the IEEE 802.11e MAC protocol service process.

Bianchi's work [17] and presented a model of the IEEE 802.11e MAC protocol for saturation throughput analysis. The IEEE 802.11e supports four classes of traffic, namely voice, video, best effort, and background traffic. Our model gives a number of performance measures including numerical results for overall network throughput and service ratio among different types of traffic, which are needed to parameterize the phase-type model for the IEEE 802.11e MAC protocol service behavior.

Our proposed phase-type model for the IEEE 802.11e MAC protocol service process is depicted in Fig. 2. In the model, $J$ is the total number of phases, and $S_{j}$ is the network state when the service phase of one packet is $j . \mu(n)$ denotes the overall service rate of the IEEE 802.11e MAC protocol given $n$ active stations and one $\mathrm{AP}$, and $\alpha(n)$ denotes the probability that a served packet is an IPTV packet. The computation of $\mu(n)$ and $\alpha(n)$ can be found in the Appendix. Note that, as shown in Fig. 2, the transition rate between neighboring phases is $J \cdot \mu(n)$ because a packet goes through $J$ phases to complete the service with a service rate of $\mu(n)$.

\section{Markovian Framework}

We develop a Markovian single server queue (SSQ) to study IPTV streaming performance over a wireless home network. In this SSQ, the birth process is modeled by the MAP and the death process is modeled by the phase-type distribution. The Markovian SSQ is described into a continuous-time Markov chain (CTMC) of five dimensional states, $\left(k_{v}, i, j, k_{p_{s}}, k_{p_{a}}\right)$, where

- $k_{v}$ is the number of video packets in the AP buffer waiting for downlink transmission, $0 \leq k_{v}<V$. The maximum buffer size in the AP for video packets is $V-1$;

- $i$ is the current phase of the IPTV video arrival in the Erlang distribution, $0 \leq i<I$, where $I$ is the total number of phases;
- $j$ is the current service phase in Erlang distribution, $0 \leq$ $j<J$, where $J$ is the total number of phases;

- $k_{p_{s}}$ is the number of stations carrying a BE packet to transmit to the $\mathrm{AP}, 0 \leq k_{p_{s}}<P S$. The maximum number of BE stations in the network is $P S-1$, which is equal to $n$;

- $k_{p_{a}}$ is the number of BE packets in the AP buffer waiting for downlink transmission, $0 \leq k_{p_{a}}<P A$. The maximum buffer size in the AP for BE packets is $P A-1$.

Tables I and II describe the one-step state transition of the birth and death transition processes of the designed SSQ, respectively. Each specified condition in the table indicates the validity of the corresponding transition to the next state with the transition rate. There are three possible events in the birth process: an advancement of an Erlang phase of an IPTV packet, an arrival of a BE packet from a station, and an arrival of a BE packet from the AP. The death process in our model corresponds to a departure of a packet or the completion of an Erlang service phase for an IPTV packet if the Erlang service phase is not the last phase. The departing packet may be a BE packet from either a station or the AP, or an IPTV packet that has completed its last Erlang service phase.

Let $x_{k_{v}, i, j, k_{p_{s}}, k_{p_{a}}}$ be the steady-state probability that the system operates in state $\left(k_{v}, i, j, k_{p_{s}}, k_{p_{a}}\right)$ and $\mathbf{x}$ be the vector holding $x_{k_{v}, i, j, k_{p_{s}}, k_{p_{a}}}$ for all possible states. The mapping of the five-dimensional state space onto a unidimensional space indexed by $q$ is achieved by

$$
\begin{aligned}
q= & i+j \cdot I+k_{v}(I \times J)+k_{p_{a}}(I \times J \times V) \\
& +k_{p_{s}}(I \times J \times V \times P A) \\
& -I \times(J-1) \times \min \left(1, k_{p_{a}}+k_{p_{s}}+k_{v}\right)
\end{aligned}
$$

where the last term is to take into account that there are only $I$ states at $k_{p_{a}}=k_{p_{s}}=k_{v}=0$. Essentially, (2) maps a fivedimension state to a unique non-negative integer number.

With the above setup, the infinitesimal generator of this CTMC can be denoted by a transition matrix $Q$, describing all the possible transitions from one state to another. With the 
TABLE II

State Transition for the Death Process From Current State $\left(k_{v}, i, j, k_{p_{s}}, k_{p_{a}}\right)$

\begin{tabular}{|c|c|c|c|}
\hline Condition & $\begin{array}{c}\text { Transition rate } \\
\text { (packets/s) }\end{array}$ & Next state & Description \\
\hline$\left[\left(k_{p_{a}}=0\right) \&\left(k_{p_{s}}=0\right) \&\left(k_{v}=1\right)\right] \&(j=0)$ & $\mu(n)$ & $(0, i, 0,0,0)$ & \multirow{3}{*}{$\begin{array}{l}\text { One packet has finished its service } \\
\text { process and no packet is waiting for } \\
\text { service. }\end{array}$} \\
\hline$\left[\left(k_{p_{a}}=0\right) \&\left(k_{p_{s}}=1\right) \&\left(k_{v}=0\right)\right] \&(j=0)$ & $\mu(n)$ & $(0, i, 0,0,0)$ & \\
\hline$\left[\left(k_{p_{a}}=1\right) \&\left(k_{p_{s}}=0\right) \&\left(k_{v}=0\right)\right] \&(j=0)$ & $\mu(n)$ & $(0, i, 0,0,0)$ & \\
\hline$\left[\left(k_{p_{a}} \geq 0\right) \&\left(k_{p_{s}}>0\right) \&\left(k_{v} \geq 0\right)\right] \&(j=0)$ & $\mu(n) \cdot(1-\alpha(n)) \cdot \frac{k_{p_{s}}}{k}$ & $\left(k_{v}, i, J-1, k_{p_{s}}-1, k_{p_{a}}\right)$ & \multirow{3}{*}{$\begin{array}{l}\text { One packet has finished its service } \\
\text { process. }\end{array}$} \\
\hline$\left[\left(k_{p_{a}}>0\right) \&\left(k_{p_{s}} \geq 0\right) \&\left(k_{v} \geq 0\right)\right] \&(j=0)$ & $\mu(n) \cdot(1-\alpha(n)) \cdot \frac{1}{k}$ & $\left(k_{v}, i, J-1, k_{p_{s}}, k_{p_{a}}-1\right)$ & \\
\hline$\left[\left(k_{p_{a}} \geq 0\right) \|\left(k_{p_{s}} \geq 0\right) \&\left(k_{v}>0\right)\right] \&(j=0)$ & $\mu(n) \cdot \alpha(n)$ & $\left(k_{v}-1, i, J-1, k_{p_{s}}, k_{p_{a}}\right)$ & \\
\hline$\left[\left(k_{p_{a}}>0\right)\left\|\left(k_{p_{s}}>0\right)\right\|\left(k_{v}>0\right)\right] \&(0<j<J)$ & $\mu(n)$ & $\left(k_{v}, i, j-1, k_{p_{s}}, k_{p_{a}}\right)$ & $\begin{array}{l}\text { One packet has transited to the next } \\
\text { Erlang service phase but yet to } \\
\text { complete the entire service process. }\end{array}$ \\
\hline
\end{tabular}

Matrix Geometric approach, this transition matrix $Q$ can be organized and partitioned into several sub-matrices as

$$
Q=\left[\begin{array}{ccccccc}
B & C & & & & \\
E & A_{1} & A_{0} & & & \\
& A_{2} & A_{1} & A_{0} & & \\
& & A_{2} & A_{1} & A_{0} & \\
& & & & & \\
& & & \ddots & \ddots & \ddots
\end{array}\right]
$$

where each of the sub-matrices, $A_{0}, A_{1}, A_{2}, B, C$, and $E$, is finite, describing the evolution of a specific state. In particular, the sub-matrix $B$ contains the state transitions among states satisfying $k_{p_{s}}=0$, and the sub-matrix $E$ contains the state transitions from any state satisfying $k_{p_{s}}=1$ to a state satisfying $k_{p_{s}}=0$. Likewise, the sub-matrix $C$ contains the state transitions from any state satisfying $k_{p_{s}}=0$ to a state satisfying $k_{p_{s}}=1$. The sub-matrix $A_{1}$ (resp. $A_{2}$ and $A_{0}$ ) contains the state transitions from any state of $k_{p_{s}}=p_{s}$ to a state satisfying $k_{p_{s}}=p_{s}$ (resp. $k_{p_{s}}=p_{s}-1$ and $\left.k_{p_{s}}=p_{s}+1\right)$.

In the $\mathrm{Q}$ matrix, each diagonal element of a row is the negative sum of all other elements at the same row. In a steady-state where transitions of arrival to and departure from a state are balanced, we establish the following system of linear equations:

$$
\mathbf{x} Q=\mathbf{0} \text {. }
$$

Given the large-scale state space of the system, solving this equation for $\mathbf{x}$ represents a challenging numerical issue. To deal with the scale of the system, we apply Neuts' Matrix-Geometric technique [19] to solve the system. In particular, we first solve

$$
A_{0}+R A_{1}+R^{2} A_{2}=\mathbf{0}
$$

where $R$ is the rate matrix, and $\mathbf{0}$ is a zero matrix with the same dimension as $A_{0}$. In the numerical computation, the problem is translated into finding the minimal non-negative solution of the matrix equation given in (5). It is performed iteratively as follows:

$$
-\left(A_{0}+R(k)^{2} A_{2}\right)\left(A_{1}\right)^{-1}=R(k+1)
$$

TABLE III

INDIVIDUAL IPTV STREAM STATISTICS

\begin{tabular}{|c|c|c|c|c|}
\hline Video & Average arrival & Average packet & \multicolumn{2}{|c|}{ Erlang parameters } \\
\cline { 4 - 5 } stream & rate (Mbps) & size (bytes) & $k$ & $\lambda_{v}$ \\
\hline ICE & 0.57 & 825.79 & 2.08 & 171.75 \\
\hline CREW & 0.87 & 926.37 & 2.12 & 260.73 \\
\hline HARBOUR & 1.72 & 954.95 & 1.72 & 356.07 \\
\hline SOCCER & 1.06 & 927.24 & 1.85 & 275.77 \\
\hline CITY & 0.93 & 908.36 & 1.81 & 242.63 \\
\hline
\end{tabular}

with $R(0)=\mathbf{0}$ and convergence determined by $\mid R(k+1)-$ $R(k) \mid<\varepsilon$, with $\varepsilon=10^{-12}$, for example.

After that, we use

$$
B[R]=\left[\begin{array}{cc}
B & C \\
E & A_{1}+R A_{2}
\end{array}\right]
$$

to solve

$$
\left(\mathbf{x}_{0}^{s}, \mathbf{x}_{1}^{s}\right) B[R]=(\mathbf{0}, \mathbf{0})
$$

with the following normalization:

$$
\mathbf{x}_{0}^{s} \mathbf{e}+\mathbf{x}_{1}^{s}(I-R)^{-1} \mathbf{e}=1
$$

to obtain $\mathbf{x}_{0}^{s}$ and $\mathbf{x}_{1}^{s}$, where $\mathbf{e}$ is the all-one vector, and $\mathbf{x}_{k_{p_{s}}}^{s}$ is the probability vector describing the network states of $k_{p_{s}}$ stations. The remaining $\mathbf{x}_{i}^{s}, i=2,3, \ldots, P S-1$ can be computed based on the following iteration using the rate matrix:

$$
\mathbf{x}_{i+1}^{s}=\mathbf{x}_{i}^{s} R
$$

The solution $\mathbf{x}$ for the entire system is obtained as

$$
\mathbf{x}=\left[\mathbf{x}_{0}^{s}, \mathbf{x}_{1}^{s}, \mathbf{x}_{2}^{s}, \ldots, \mathbf{x}_{P S-1}^{s}\right]
$$

Based on the Matrix-Geometric solution, we can obtain the steady-state probability distribution for streaming TV over the IEEE 802.11e wireless home network, and the sum of the steady-state probability distribution under no packet loss condition can be used to determine the throughput of IPTV $T_{n}$ in the $n$-station wireless home network with $n$ IPTV flows. The throughput of the IPTV traffic $T_{n}$ in the $n$-station network can be computed as

$$
T_{n}=r_{v} \cdot \sum_{k_{p_{s}}=0}^{P S-1} \sum_{k_{v}=0}^{V-2} \sum_{k_{p_{a}}=0}^{P A-1} \sum_{i=0}^{I-1} \sum_{j=0}^{J-1} x_{k_{v}, i, j, k_{p_{s}}, k_{p_{a}}}
$$


TABLE IV

AGGREGATED IPTV TRAFFIC STATISTICS

\begin{tabular}{|c|c|c|c|c|c|}
\hline \multirow{2}{*}{$\begin{array}{c}\text { Number of } \\
\text { IPTV streams }\end{array}$} & \multirow[t]{2}{*}{ Mix of video streams } & \multirow{2}{*}{$\begin{array}{l}\text { Average arrival } \\
\text { rate }(\mathrm{Mbps})\end{array}$} & \multirow{2}{*}{$\begin{array}{l}\text { Average packet } \\
\text { size (bytes) }\end{array}$} & \multicolumn{2}{|c|}{ Erlang parameters } \\
\hline & & & & $k$ & $\lambda_{v}$ \\
\hline 1 & $1 \mathrm{ICE}$ & 0.57 & 825.79 & 2.08 & 171.75 \\
\hline 2 & $1 \mathrm{ICE}+1 \mathrm{CREW}$ & 1.21 & 876.08 & 2.10 & 373.29 \\
\hline 3 & $1 \mathrm{ICE}+1$ CREW + 1 HARBOUR & 2.64 & 901.67 & 1.32 & 496.47 \\
\hline 4 & 1 ICE +1 CREW + 1 HARBOUR + 1 SOCCER & 3.36 & 908.58 & 2.05 & 973.37 \\
\hline 5 & 1 ICE +1 CREW + 1 HARBOUR + 1 SOCCER + 1 CITY & 4.14 & 908.54 & 2.04 & 1205.50 \\
\hline 6 & 2 ICE +1 CREW + 1 HARBOUR +1 SOCCER +1 CITY & 4.51 & 894.75 & 1.99 & 1293.19 \\
\hline 7 & 2 ICE + 2 CREW + 1 HARBOUR + 1 SOCCER + 1 CITY & 5.23 & 899.27 & 1.94 & 1453.07 \\
\hline 8 & 2 ICE +2 CREW + 2 HARBOUR + 1 SOCCER + 1 CITY & 6.66 & 906.23 & 1.81 & 1717.05 \\
\hline 9 & 2 ICE +2 CREW +2 HARBOUR +2 SOCCER +1 CITY & 7.37 & 908.56 & 2.05 & 2146.96 \\
\hline 10 & $2 \mathrm{ICE}+2 \mathrm{CREW}+2$ HARBOUR +2 SOCCER +2 CITY & 8.16 & 908.54 & 1.89 & 2189.60 \\
\hline 11 & $3 \mathrm{ICE}+2$ CREW +2 HARBOUR +2 SOCCER +2 CITY & 8.53 & 918.30 & 1.94 & 2365.95 \\
\hline 12 & 3 ICE +3 CREW +2 HARBOUR + 2 SOCCER +2 CITY & 9.25 & 919.02 & 2.15 & 2838.83 \\
\hline 13 & 3 ICE +3 CREW +3 HARBOUR +2 SOCCER +2 CITY & 10.68 & 923.58 & 2.06 & 3129.60 \\
\hline 14 & 3 ICE +3 CREW +3 HARBOUR +3 SOCCER +2 CITY & 11.40 & 923.80 & 2.00 & 3246.43 \\
\hline 15 & 3 ICE +3 CREW + 3 HARBOUR + 3 SOCCER + 3 CITY & 12.18 & 922.82 & 2.09 & 3617.57 \\
\hline
\end{tabular}

where $r_{v}$ is defined in (1). Equation (12) is basically computing the mean of IPTV arrival rate. In a lossless system, the number of arrivals is equal to the number departures, i.e., the throughput.

\section{Performance Evaluation}

In this section, we measure several key performance metrics of IPTV streaming over wireless home networks. Using our developed model, we first measure the system capacity highlighting the potential system support of the existing and future wireless home networks. We further perform network simulator (ns2) [20] simulations with real video streams [21] so as to validate our numerical results, and measure various important system and streaming performance including the channel capacity, packet delay and jitter, instant, and average PSNR behavior of IPTV streaming.

We use five different 4 CIF video sources, ${ }^{1}$ ICE, CREW, HARBOUR, SOCCER, and CITY, to generate the test IPTV streams. Each video source is of a resolution of $704 \times 576$, and is compressed using H.264/AVC JSVM 9.8 [22] with parameters of $30 \mathrm{fps}$, a GoP of 16 frames, the quantization parameters (QPs) of 32, 34 and 35 for I, P, and B frames, respectively. The statistics parameters of the five video streams are shown in Table III.

To generate an aggregated IPTV traffic of $n$ IPTV streams, we recursively select streams from the set of the ordered five video streams \{ICE, CREW, HARBOUR, SOCCER, and CITY $\}$ and each stream begins at a random start time within the first $0.5 \mathrm{~s}$ of the experiment. By mixing different video streams, we simulate the common scenario that different home users watch different types of TV programs. Of course, the five test video streams can be ordered in other ways. Table IV shows the statistics of the generated aggregated IPTV traffic with different numbers of IPTV streams.

We assume the presence of a certain amount of BE traffic sent between the stations and AP. We use Poisson process [23] to model the $\mathrm{BE}$ traffic. While the default arrival rates, $\lambda_{p_{a}}$ and $\lambda_{p_{s}}$, for the downlink and uplink BE traffic are set to 10 packets/s and 20 packets/s, respectively, with a packet size of 1024 bytes, we also consider various levels of BE traffic load.

${ }^{1}$ http://nsl.cs.sfu.ca/wiki/index.php/Video Library and Tools
Other parameters, I, J, PA, and V, in the proposed Markovian framework, are set to $3,3,3$, and 10 , respectively.

We study a wireless home network that implements the QoS enhancement specified in the IEEE 802.11e standard. In terms of channel data rate, we model both the IEEE $802.11 \mathrm{~b}$ standard and the IEEE $802.11 \mathrm{~g}$ standard operating at $11 \mathrm{Mbps}$ and 54 Mbps, respectively. Moreover, since our model allows arbitrary channel data rate settings, we also consider scenarios of future wireless home networks where the wireless channel operates at a much higher data rate ${ }^{2}$ such as 100 Mbps.

\section{A. System Capacity}

We first measure the system capacity in terms of the number of IPTV streams that can be supported by a wireless home network using either the IEEE $802.11 \mathrm{~b}$, the IEEE $802.11 \mathrm{~g}$, or the IEEE $802.11 \mathrm{n}$ standard. We show the throughput under different number of IPTV streams in Fig. 3. Numerical results are obtained by solving the corresponding CTMC using the Matrix-Geometric approach. Simulation results obtained by ns 2 are included in the figure for comparison. The excellent agreement between the numerical and simulation results confirms the accuracy of our model.

It can be seen from Fig. 3 that the IEEE $802.11 \mathrm{~b}$ standard can merely support the two IPTV streams (the aggregation of ICE and CREW shown in Table IV) and the channel becomes congested when the three IPTV streams in Table IV are transmitted. Thus, it has very limited potential to prepare for future advancements in either supporting more simultaneous IPTV streams or higher quality streams. The IEEE $802.11 \mathrm{~g}$ standard, on the other hand, has significant improvement in the system capacity, supporting the six IPTV streams. While the channel data rate increases almost five times from $11 \mathrm{Mbps}$ of IEEE $802.11 \mathrm{~b}$ to 54 Mbps IEEE $802.11 \mathrm{~g}$, the capacity support of IPTV streams, which form the majority of the traffic, increases less than four times. This suggests that the system capacity of IPTV does not

\footnotetext{
${ }^{2}$ Note that when considering future higher data rate, we adopt the current practice in the standard development that future IEEE 802.11 standards are backward compatible with the existing IEEE 802.11 standard. For example, we consider that future IEEE 802.11 standards operating at $2.4 \mathrm{GHz}$ must follow the existing IEEE $802.11 \mathrm{~b}$ standard to transmit a packet, except that the transmission of the payload within a packet may be involved in an advanced transmission technology that will result in a shorter transmission duration.
} 


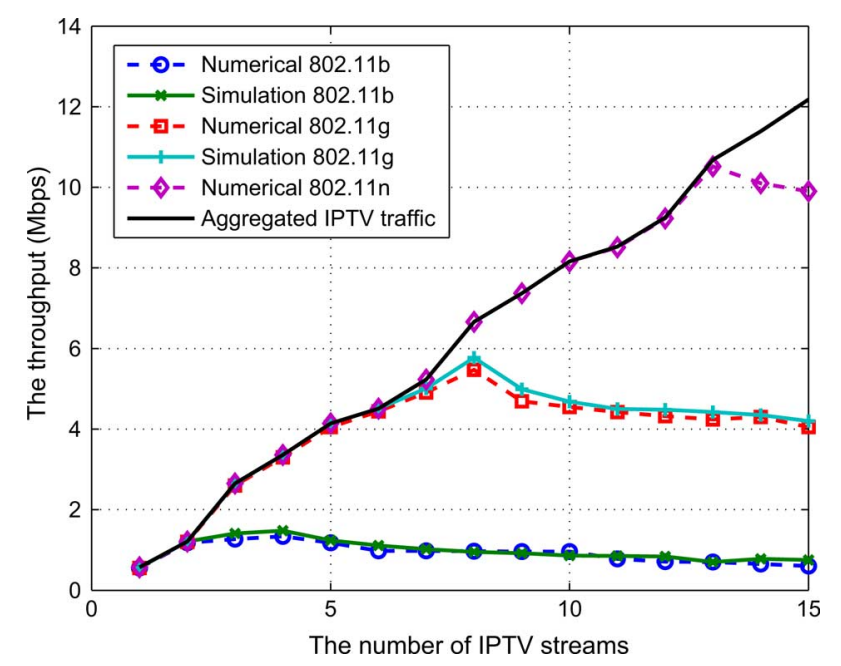

Fig. 3. IPTV throughput of different wireless home networks with default light $\mathrm{BE}$ traffic.

grow linearly with the data rate, which is consistent with earlier reports.

The observation of nonlinear growth of the system capacity with the channel data rate has prompted the investigation of system capacity study for future wireless home network with higher channel data rates. ${ }^{3}$ From Fig. 3 , we can see that for new emerged wireless communications technologies operating at 2.4 $\mathrm{GHz}$ offering $100 \mathrm{Mbps}$, the system supports 13 simultaneous IPTV streams for the aggregated traffic specified in Table IV. The results suggest that the very recent development of the IEEE $802.11 \mathrm{n}$ standard operating at $100 \mathrm{Mbps}$ already offers adequate support for the IPTV application.

Next, we investigate the impact of BE traffic on the system capacity. We vary the overall $\mathrm{BE}$ traffic load from $30 \mathrm{kbps}$ to 2.64 Mbps, where the downlink BE traffic and the uplink BE traffic are given one third and two third of the load, respectively. Fig. 4 shows the IPTV throughput under different overall BE load. From the figure, it can be seen that in the case of streaming the two IPTV streams over the IEEE $802.11 \mathrm{~b}$ wireless home network, as the offered load of the BE traffic reaches around $1.31 \mathrm{Mbps}$, the transmission collision intensifies and the IPTV throughput drops below a level to adequately delivery the two simultaneous IPTV streams. Likewise, in the case of streaming the six IPTV streams over the IEEE 802.11g wireless home network, a drop trend of the IPTV throughput is developed at around $0.47 \mathrm{Mbps}$ of the BE traffic load. These results tell that while the IEEE 802.11e standard specifies priority delivery for video traffic, due to its contention-based design, the $\mathrm{BE}$ traffic can affect the delivery of IPTV video streams.

\section{B. Delay Performance}

Fig. 5(a) shows individual packet delay of the ICE video stream in the cases of streaming the two streams and the three streams specified in Table IV over IEEE $802.11 \mathrm{~b}$. Here, the delay measures the interval from the time when a packet arrives to the AP from the Internet to the time when the packet reaches

\footnotetext{
${ }^{3}$ Such developments are already underway with IEEE $802.11 \mathrm{n}$ for $100 \mathrm{Mbps}$.
}

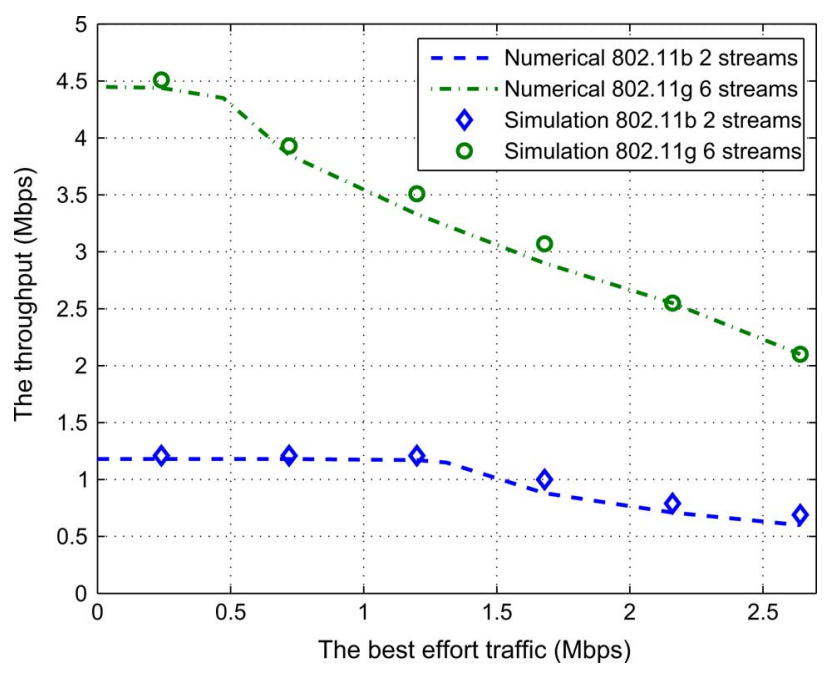

Fig. 4. IPTV throughput of the IEEE $802.11 \mathrm{~b}$ and the IEEE $802.11 \mathrm{~g}$ wireless home networks for a range of intensity of BE traffic.

the intended station. The limitation of the IEEE $802.11 \mathrm{~b}$ standard for IPTV application is clearly illustrated. While the video packets suffer over 300-ms delay in the AP showing the inadequate of support of the IEEE $802.11 \mathrm{~b}$ wireless home networks with the three simultaneous IPTV streams, the network appears to cope well in the case of streaming the two IPTV streams. We further show the cumulative distribution of delay and jitter of streaming the two IPTV streams in Fig. 6(a). As can be seen, $95 \%$ of the IPTV packets are served by the AP within $20 \mathrm{~ms}$, and $94 \%$ of packet jitter is within $10 \mathrm{~ms}$.

Fig. 5(b) shows individual packet delay of the ICE video stream over the time for the IEEE $802.11 \mathrm{~g}$ wireless home network. We see that with a higher channel data rate, the wireless home network can now carry up to six simultaneous IPTV streams. With adding in the seventh IPTV stream, there is a sharp increase in packet delay. In Fig. 6(b), we show the cumulative distribution of packet delay and jitter for the case of streaming the six IPTV streams. The results report that almost all IPTV packets are served by the AP within $20 \mathrm{~ms}$ with delay jitter within $10 \mathrm{~ms}$.

\section{PSNR Performance}

Fig. 7 shows the PSNR performance of the ICE video stream in both the IEEE $802.11 \mathrm{~b}$ and the IEEE $802.11 \mathrm{~g}$ wireless home networks operating at the critical points. The results further confirm that the IEEE $802.11 \mathrm{~b}$ and the IEEE $802.11 \mathrm{~g}$ wireless home networks can support the specified two and six IPTV streams, respectively, achieving an average PSNR of over $35 \mathrm{~dB}$. With one more IPTV stream adding to the networks, the PSNR performance of the ICE video stream drops sharply to around 15 $\mathrm{dB}$ in both of the networks.

Furthermore, given a number of simultaneous IPTV streams, we compute the average PSNR observed at all receivers. Fig. 8 shows the average PSNR performances for various number of simultaneous IPTV streams over the IEEE $802.11 \mathrm{~b}$ and $802.11 \mathrm{~g}$ wireless home networks. Again, we observe that satisfactory average PSNR performance is achieved with the two and six IPTV 


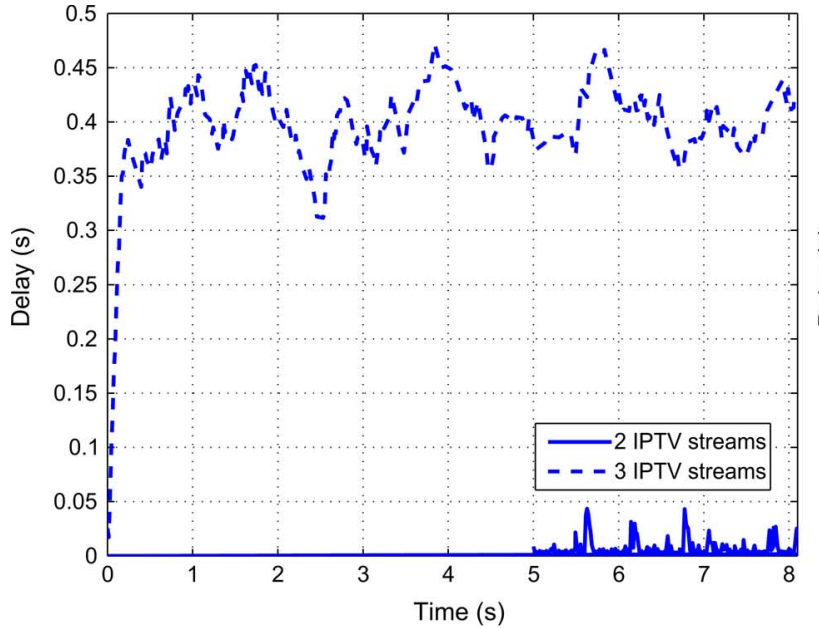

(a)

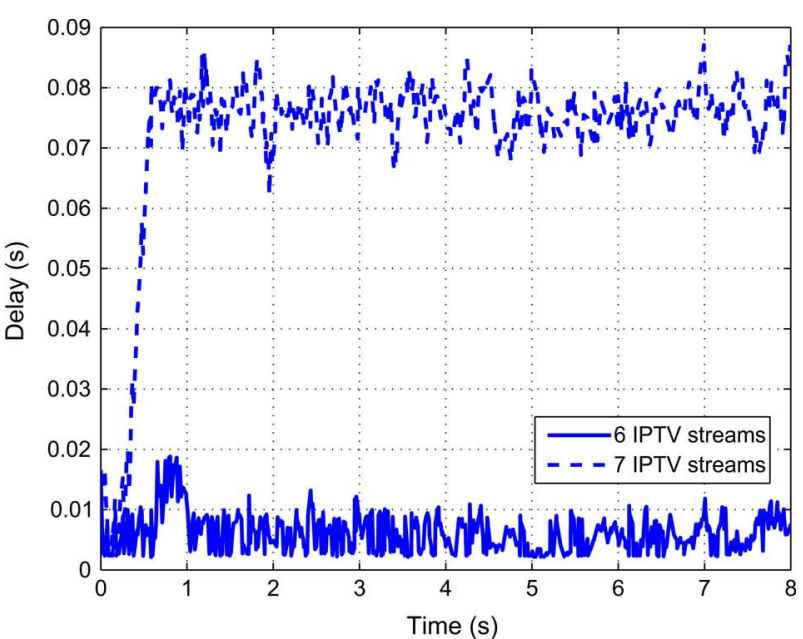

(b)

Fig. 5. Packet delay of the ICE video stream. (a) IEEE 802.11b. (b) IEEE 802.11g.

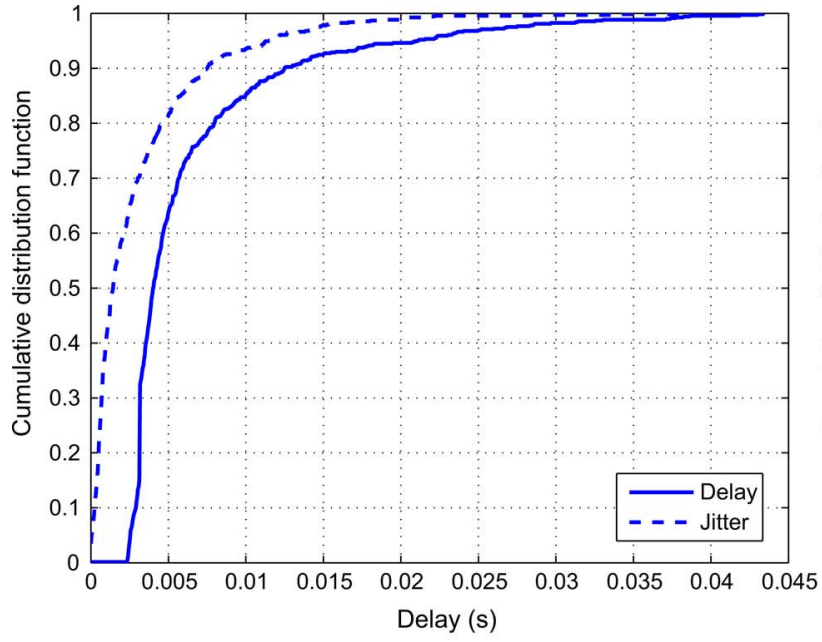

(a)

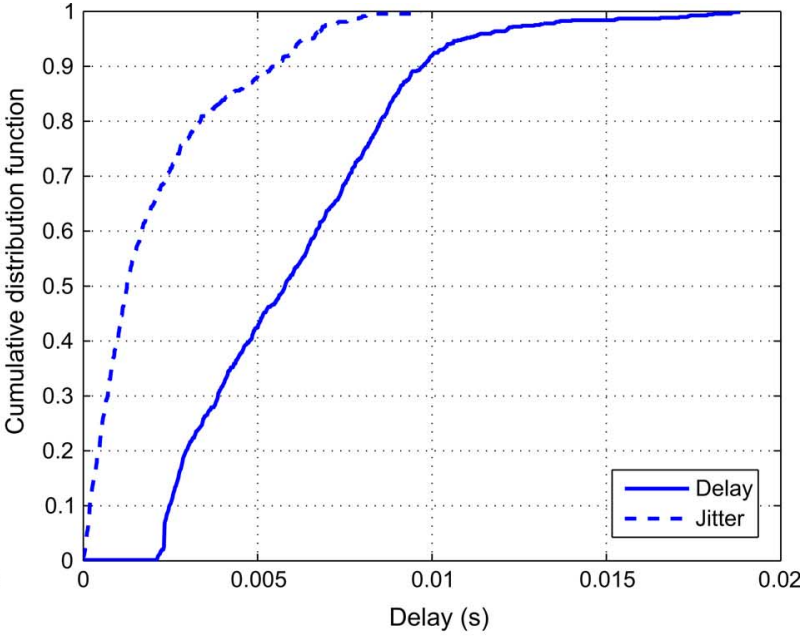

(b)

Fig. 6. Cumulative distribution function of packet delay and jitter of the ICE video stream. (a) IEEE 802.11b with two IPTV streams. (b) IEEE 802.11g with six IPTV streams.

streams for the IEEE $802.11 \mathrm{~b}$ and IEEE $802.11 \mathrm{~g}$ networks, respectively. When the number of IPTV streams increases, the average PSNR quickly drops indicating inadequate support. This is consistent with the capacity result illustrated in Fig. 3.

\section{CONCLUSIONS}

In this paper, we studied the performance of IPTV streaming over wireless home networks. We developed a Markovian framework to model the IPTV traffic streams and the IEEE 802.11e MAC protocol. With the developed model, we investigated the system capacity, delay and jitter, and PSNR performance. Additionally, we performed simulation experiments using ns2 to validate our numerical results and further study the performance in other aspects.

Our investigation shows that in general, the IEEE $802.11 \mathrm{~b}$ standard is insufficient to support the IPTV application while the IEEE $802.11 \mathrm{~g}$ standard is suitable. For the particular scenario we test, we show that IEEE $802.11 \mathrm{~b}$ can only support up to two simultaneous IPTV streams while IEEE $802.11 \mathrm{~g}$ supports up to six simultaneous IPTV streams. After factoring surrounding interference, distanced connection with a lower physical data rate, and higher quality, we expect that the IEEE $802.11 \mathrm{~b}$ standard will soon appear as a bottleneck. As the demand for highquality streaming increases, a higher speed wireless home network, such as the IEEE 802.11n standard, is required.

Besides, our study also confirms that the system capacity of IPTV in a wireless home network does not grow linearly with the data rate. While increasing the data rate of a wireless home network may represent an effective solution to increase the system capacity of IPTV, other efforts such as an efficient MAC protocol operation and QoS mechanism may also be necessary to further improve the network performance in order to achieve the future vision of pervasive personal entertainment enjoyment. 


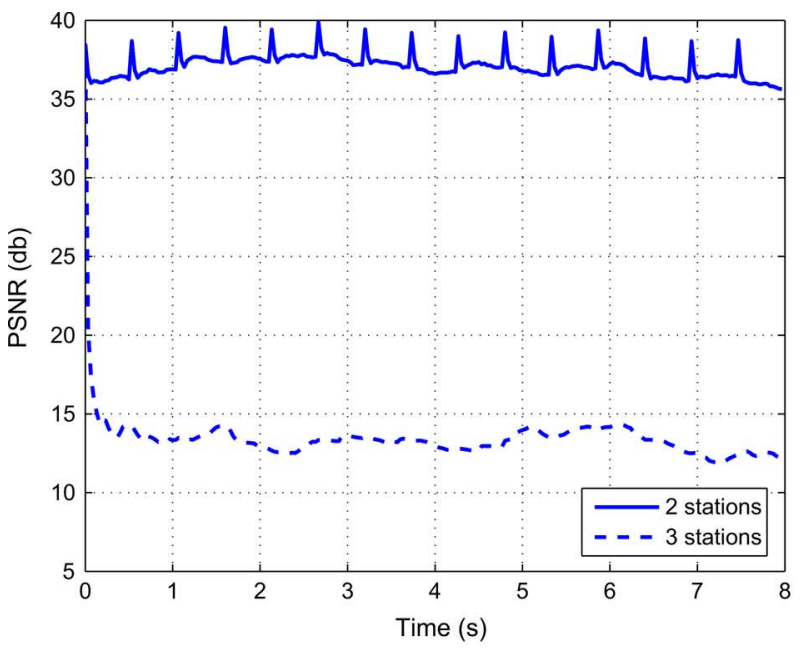

(a)

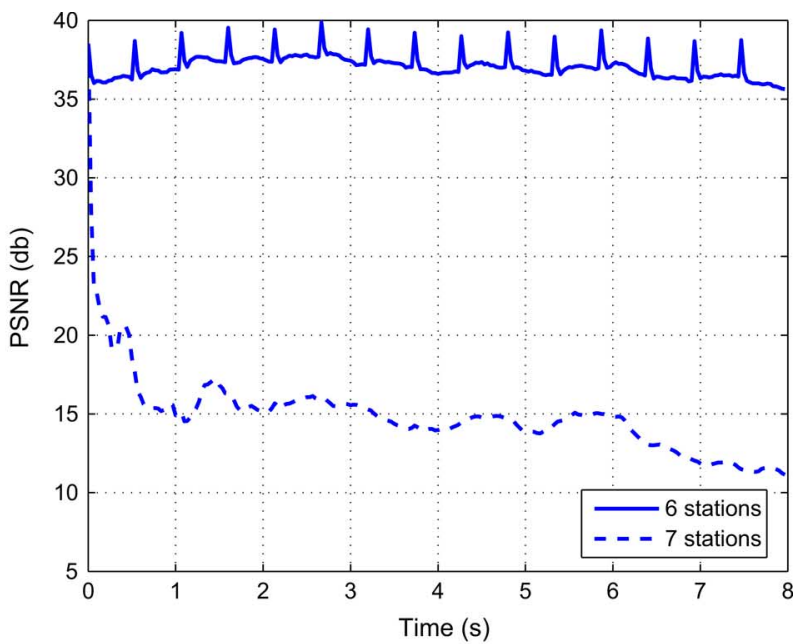

(b)

Fig. 7. PSNR performance of the ICE video stream. (a) IEEE 802.11b. (b) IEEE 802.11g.

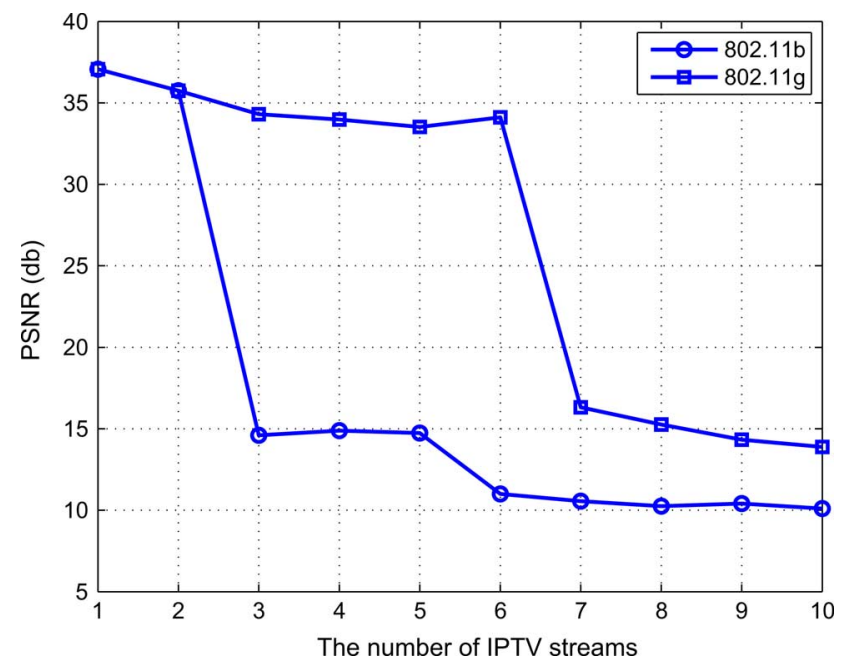

Fig. 8. Average PSNR performance in the IEEE 802.11b and IEEE 802.11g wireless home network with default light BE traffic.

TABLE V

MAC PROTOCOL SYSTEM PARAMETERS

\begin{tabular}{|c|c|c|c|c|}
\hline $\begin{array}{c}\text { Access } \\
\text { category }\end{array}$ & AIFS & $C W_{\min }$ & $\begin{array}{c}\text { Maximum } \\
\text { retry limit, } r\end{array}$ & $\begin{array}{c}\text { Maximum } \\
\text { backoff stage, } m\end{array}$ \\
\hline IPTV & 2 & 7 & 7 & 4 \\
\hline BE & 7 & 31 & 7 & 5 \\
\hline
\end{tabular}

APPENDIX

\section{IEEE 802.11E SERVICE MODEL}

In this Appendix, we show how to compute the channel service rate, $\mu(n)$, and the video service ratio, $\alpha(n)$. We assume that there are $n$ IPTV flows and there exists BE traffic to be delivered in both the AP and the $n$ stations. The MAC parameters for IPTV traffic and the BE traffic are shown in Table V.

We consider access categories $\left(A C_{c}\right)$ of traffic, $c \in$ $\{\mathrm{IPTV}$ or BE\}, and the links of a certain access category $(A C)$ maintains its persistence probability, $\tau_{c}$, to access the channel in each time-slot. We model the backoff operation of each station with a Markov chain. This model is based on the
Bianchi's work [17] with the addition of frame retransmission limit. The state $i, j$ corresponds to the $i \mathrm{TH}$ backoff stage of a station and that station has $j$ as its backoff counter. The variable $i$ ranges from 0 as the first backoff stage to $r_{c}$ as the retransmission limit. The value $j$ ranges from 0 to $W_{c, i}-1$, where $W_{c, i}$ is the backoff window of stage $i$ in $A C_{c}$; the backoff window $W_{c, i}$ is given by

$$
W_{c, i}= \begin{cases}2^{i} \cdot C W_{c, \min }, & 0 \leq i \leq m_{c} \\ 2^{m_{c}} \cdot C W_{c, \min } & m_{c}<i \leq r_{c} .\end{cases}
$$

Let $p_{c}$ denote the conditional collision probability, which is the probability that a frame encounters a collision when it is transmitted. Let $\alpha_{c, i, j}$ be the stationary distribution for $A C_{c}$. Owing to the chain regularities, we have

$$
\begin{aligned}
& \alpha_{c, i, 0}=p_{c} \cdot \alpha_{c, i-1,0}, \quad 0<i \leq r_{c, i} \\
& \alpha_{c, i, j}=\frac{W_{c, i}-j}{W_{c, i}} \cdot \alpha_{c, i, 0}, \quad 0 \leq i \leq r_{c, i}, 0<j<W_{c, i} .
\end{aligned}
$$

Imposing the normalization condition, $\sum_{i, j} \alpha_{c, i, j}=1$, we obtain

$$
\alpha_{c, 0,0}= \begin{cases}\frac{\xi_{c}}{\iota_{c, r}+\kappa_{c}}, & r_{c} \leq m_{c} \\ \frac{\xi_{c}}{\iota_{c, m}+\kappa_{c}+\nu_{c}}, & r_{c}>m_{c}\end{cases}
$$

where

$$
\begin{aligned}
\xi_{c} & =2\left(1-2 p_{c}\right)\left(1-p_{c}\right) \\
\iota_{c, r} & =W_{c, 0}\left(1-\left(2 p_{c}\right)^{r_{c}+1}\right)\left(1-p_{c}\right) \\
\iota_{c, m} & =W_{c, 0}\left(1-\left(2 p_{c}\right)^{m_{c}+1}\right)\left(1-p_{c}\right) \\
\kappa_{c} & =\left(1-2 p_{c}\right)\left(1-p_{c}^{r_{c}+1}\right) \\
\nu_{c} & =W_{c, 0} 2^{m_{c}} p_{c}^{m_{c}+1}\left(1-2 p_{c}\right)\left(1-p_{c}^{r_{c}-m_{c}}\right)
\end{aligned}
$$

and $n_{c}$ denotes the number of stations belonging to different ACs, $r_{c}$ and $m_{c}$ are the maximum retry limit and the maximum backoff stage, respectively, and $W_{c, 0}$ is the minimum backoff window size for $A C_{c}$. The maximum backoff stage describes the maximum number that the contention window may double 
counting from $C W_{\min }$, and this number is capped at the retry limit.

The probability $\tau_{c}$ that a station of $A C_{c}$ transmits in a slot time is given by

$$
\tau_{c}=\sum_{i=0}^{r_{c}} \alpha_{c, i, 0}=\frac{1-p_{c}^{r_{c}+1}}{1-p_{c}} \alpha_{c, 0,0}
$$

The probability $\tau_{c}$ depends on the collision probability $p_{c}$ which is given by

$$
p_{c}=1-\left(1-\tau_{c}\right)^{n_{c}-1} \prod_{x \neq c}\left(1-\tau_{c}\right)^{n_{x}} .
$$

Thus, the throughput is given by

$$
S_{c}=\frac{P_{c, S} T[P]_{c}}{T[S T]}
$$

where $P_{c, S}$ is the probability that a slot contains a successful transmission which is given by

$$
P_{c, S}=n_{c} \tau_{c}\left(1-\tau_{c}\right)^{n_{c}-1} \prod_{x \neq c}\left(1-\tau_{c}\right)^{n_{x}} .
$$

The probability that a slot time is idle is $P_{I}, P_{I}=\sum_{c}(1-$ $\left.\tau_{c}\right)^{n_{c}}$. The probabilities $P_{C}$ that a slot time contains a collision is $P_{C}=1-P_{I}-P_{S}$, where $P_{S}=\sum_{c} P_{c, S} . T[P]_{c}$ is the average time length of the frame payload of $A C_{c}$. It depends on the channel data rate $R$ and the frame payload size $L_{c} . T[S T]$ is the average length of a time slot and the value $T[S T]$ is computed by $T[S T]=P_{I} \sigma+P_{S} T_{S}+P_{C} T_{C}$, where $\sigma$ is the length of a slot time, $T_{S}$ is the average length of a successful transmission slot, and $T_{C}$ is the average length of a collision slot. Their numerical values can be obtained based on the computation given in [18].

Finally, the $\alpha(n)$ can be calculated by

$$
\alpha(n)=\frac{S_{I P T V}(n)}{S_{I P T V}(n)+S_{B E}(n)} .
$$

The service rate $\mu_{c}(n)$, depending on the channel data rate and the frame payload size, can be computed as $\mu(n)=\sum_{c} \mu_{c}(n)$ (packets/s), where

$$
\mu_{c}(n)=\frac{R \cdot S_{c}(n)}{L_{c}}
$$

[5] N. Nasser, "Service adaptability in multimedia wireless networks," IEEE Trans. Multimedia, vol. 11, no. 4, pp. 786-792, Jun. 2009.

[6] J. Hu, S. Choudhury, and J. Gibson, "Video capacity of WLANs with a multiuser perceptual quality constraint," IEEE Trans. Multimedia, vol. 10, no. 8, pp. 1465-1478, Dec. 2008.

[7] K. Ahmad and A. Begen, "IPTV and video networks in the 2015 timeframe: The evolution to medianets," IEEE Commun. Mag., vol. 47, no. 12, pp. 68-74, Dec. 2009.

[8] C. Foh, M. Zukerman, and J. Tantra, "A Markovian framework for performance evaluation of IEEE 802.11," IEEE Trans. Wireless Commun., vol. 6, no. 4, pp. 1276-1265, Apr. 2007.

[9] I. Gerhardt and B. L. Nelson, "Transforming renewal processes for simulation of nonstationary arrival processes," INFORMS J. Comput., vol. 21, no. 4, pp. 630-640, 2009.

[10] Y. Xiao, X. Du, J. Zhang, and F. Hu, "Internet protocol television (IPTV): The killer application for the next-generation Internet," IEEE Commun. Mag., vol. 45, no. 11, pp. 126-134, Nov. 2007.

[11] I. Djama and T. Ahmed, "A cross-layer interworking of DVB-T and WLAN for mobile IPTV service delivery," IEEE Trans. Broadcast., vol. 53, no. 1, pt. 2, pp. 382-390, Mar. 2007.

[12] Q. Du and X. Zhang, "Statistical QoS provisionings for wireless unicast/multicast of layered video streams," in Proc. INFOCOM, 2009, pp. 477-485.

[13] E. Shihab, F. Wan, L. Cai, A. Gulliver, and N. Tin, "Performance analysis of IPTV traffic in home networks," in Proc. IEEE Global Telecommunications Conf., 2007, pp. 5341-5345.

[14] E. Shihab, L. Cai, F. Wan, A. Gulliver, and N. Tin, "Wireless mesh networks for in-home IPTV distribution," IEEE Network, vol. 22, no. 1, pp. 52-57, Jan./Feb. 2008.

[15] D. Li and J. Pan, "Performance evaluation of video streaming over multi-hop wireless local area networks," IEEE Trans. Wireless Commun., vol. 9, no. 1, pp. 338-347, Jan. 2010.

[16] P. Symes, Digital Video Compression. New York: McGrawHill/TAB Electronics, 2004.

[17] G. Bianchi, "Performance analysis of the IEEE 802.11 distributed coordination function," IEEE J. Select Areas Commun., vol. 18, no. 3, pp. 535-547, Mar. 2000.

[18] J. Tantra, C. Foh, and A. Mnaouer, "Throughput and delay analysis of the IEEE 802.11e EDCA saturation," in Proc. IEEE Int. Conf. Communications, 2005, vol. 5 .

[19] M. Neuts, Matrix-Geometric Solutions in Stochastic Models. Baltimore, MD: Johns Hopkins Univ. Press, 1981.

[20] NS-2 Network Simulator. [Online]. Available: http://www.isi.edu/ nsnam $/ \mathrm{ns} /$.

[21] C.-H. Ke, C.-K. Shieh, W.-S. Hwang, and A. Ziviani, "An evaluation framework for more realistic simulations of MPEG video transmission," J. Inf. Sci. Eng., vol. 24, no. 2, pp. 425-440, 2008.

[22] J. Reichel, H. Schwarz, and M. Wien, "JSVM 9.8 software," in Joint Video Team of ISO/IEC MPEG and ITU-T VCEG, 2007, vol. 9212.

[23] S. Mangold, S. Choi, P. May, O. Klein, G. Hiertz, and L. Stibor, "IEEE 802.11e wireless LAN for quality of service," Eur. Wireless, pp. 32-39, Feb. 2002.

\section{REFERENCES}

[1] Wireless LAN Medium Access Control (MAC) and Physical Layer (PHY) Specifications: Higher-Speed Physical Layer Extension in the $2.4 \mathrm{GHz}$ Band, IEEE Std. 802.11b, 2000.

[2] IEEE 802.11g-2003: Further Higher Data Rate Extension in the 2.4 GHz Band, IEEE Std. 802.11g, Sep. 2007.

[3] Wireless LAN Medium Access Control (MAC) and Physical Layer (PHY) specifications Amendment 8: Medium Access Control (MAC) Quality of Service Enhancements, IEEE Std. 802.11e, 2005.

[4] IEEE 802.11n-2009: Amendment 5: Enhancements for Higher Throughput, IEEE Std. 802.11n, Oct. 2009.

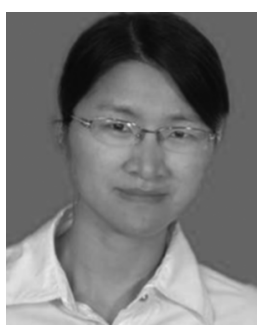

Tiantian Guo (M'06) received the B.Eng. degree in electrical engineering from Beijing University of Aeronautics and Astronautics, Beijing, China, in 2004 and the M.Eng. degree in biomedical engineering from Beijing University of Posts and Telecommunications in 2006. She is currently pursuing the Ph.D. degree in the School of Computer Engineering, Nanyang Technological University, Singapore.

Her research interests are in the area of mobile and and cross-layer protocol design.

wireless networks with emphasis on QoS guarantee 


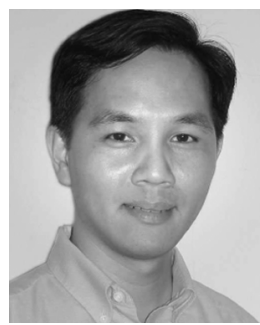

Chuan Heng Foh (S'00-M'03-SM'09) received the $\mathrm{Ph} . \mathrm{D}$. degree from the University of Melbourne, Melbourne, Australia, in 2002.

From July 2002 to December 2002, he was a Lecturer at Monash University, Melbourne. In December 2002, he joined the School of Computer Engineering, Nanyang Technological University, Singapore, as an Assistant Professor. His research interests include protocol design and performance analysis of mobile wireless and sensor networks.

Dr. Foh is serving on the editorial board of the $I n-$ ternational Journal of Communications Systems.

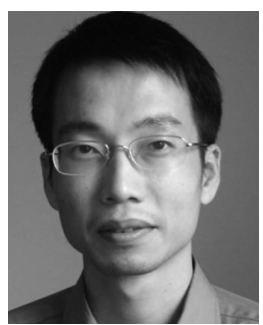

Jianfei Cai (S'98-M'02-SM'07) received the Ph.D. degree from the University of Missouri-Columbia in 2002.

Currently, he is an Associate Professor and also the Head of the Computer Communications Division at the School of Computer Engineering, Nanyang Technological University, Singapore. His major research interests include visual signal processing and multimedia networking. He has published more than 100 technical papers in international conferences and journals. He has actively participated in program committees of various conferences.

Dr. Cai served as one of the track co-chairs for IEEE ICME 2006 \& 2008 \& 2009, the technical program co-chair for Multimedia Modeling (MMM) 2007, and the conference co-chair for Multimedia on Mobile Devices 2007. He will serve as a technical program chair for ICME 2012. He is also an Associate Editor for the IEEE TRANSACTIONS ON CIRCUITS AND SYSTEMS FOR VIDEO TECHNOLOGY (T-CSVT).

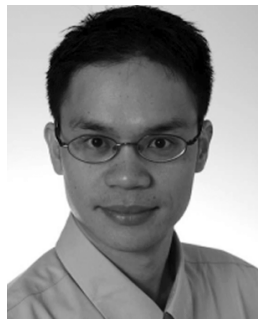

Dusit Niyato (M'09) received the B.E. degree from King Mongkut's Institute of Technology Ladkrabang (KMITL), Bangkok, Thailand, in 1999 and the Ph.D. degree in electrical and computer engineering from the University of Manitoba, Winnipeg, MB, Canada, in 2008 .

He is currently an Assistant Professor in the Division of Computer Communications, School of Computer Engineering, Nanyang Technological University, Singapore. His current research interests include design, analysis, and optimization of wireless communication, smart grid systems, green radio communications, and mobile cloud computing.

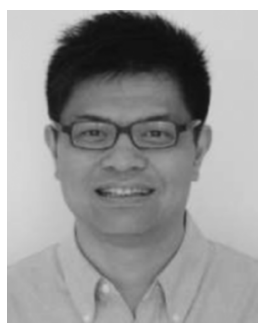

Eric W. M. Wong (S'87-M'90-SM'00) received the B.Sc. and M.Phil. degrees in electronic engineering from the Chinese University of Hong Kong, Hong Kong, in 1988 and 1990, respectively, and the Ph.D. degree in electrical and computer engineering from the University of Massachusetts, Amherst, in 1994.

In 1994, he joined the City University of Hong Kong, where he is now an Associate Professor with the Department of Electronic Engineering. His research interests include the analysis and design of telecommunications networks, optical switching, and video-on-demand systems. His most notable research work involved the first accurate and workable model for state-dependent dynamic routing based on the Erlang fixed-point approximation presented in Infocom'90. Since 1991, the model has been used by AT\&T to design and dimension its telephone network that uses real-time network routing. 